Rev. Bras. Saúde Prod. Anim., Salvador, v.16, n.1, p.223-231 jan./mar., 2015 http://www.rbspa.ufba.br ISSN 15199940

\title{
Inclusão da farinha do mesocarpo de babaçu em rações de frangos de corte na fase de crescimento/terminação ( 22 a 42 dias)
}

\author{
Inclusion ofmesocarpbabassu flour in broilers diets in growing/finishing stage (22 the \\ 42 days old)
}

\author{
CRUZ, Lília Alves da ${ }^{2} *$; SIQUEIRA, Jefferson Costa de ${ }^{4}$;VAZ, Roberta Gomes Marçal \\ Vieira $^{3}$;RODRIGUES, Kênia Ferreira ${ }^{3}$; SILVA, Mônica Calixto da ${ }^{3}$; FONSECA, Flávia \\ Luzia Rodrigues $^{3}$;PARENTE, Iberê Pereira ${ }^{3}$; ALVES, Carla Fonseca ${ }^{3}$; SILVA, Ariane \\ de Sousa Rodrigues ${ }^{5}$
}

\author{
${ }^{1}$ Parte da Dissertação de Mestrado do primeiro autor. \\ ${ }^{2}$ Zootecnista Extensionista Ruraltins, Paraíso, Tocantins, Brasil. \\ ${ }^{3}$ Universidade Federal do Tocantins, Escola de Medicina Veterinária e Zootecnia, Araguaína, Tocantins, Brasil. \\ ${ }^{4}$ Universidade Federal do Maranhão, Centro de Ciências Agrárias e Ambientais, Chapadinha, \\ Maranhão, Brasil. \\ ${ }^{5}$ Zootecnista, Autônoma, Araguaína, Tocantins, Brasil. \\ *Endereço para correspondência: lilia.al.cruz@ hotmail.com
}

\section{RESUMO}

O trabalho foi desenvolvido com o objetivo de avaliar a viabilidade técnica e econômica da inclusão da farinha do mesocarpo do babaçu, em dietas de frangos de corte de 22 a 42 dias de idade. Foram utilizados 80 frangos de corte, machos, da linhagem Cobb $500^{\circledR}$, distribuídos em delineamento experimental inteiramente casualizado com quatro tratamentos $(0 ; 6 ; 12 \mathrm{e}$ $18 \%$ de inclusão da farinha do mesocarpo de babaçu)e cinco repetições, sendo cada parcela experimental composta por 4 aves. Aos 42 dias de idade as aves e as rações experimentais foram pesadas para obtenção do desempenho (consumo de ração, ganho peso e conversão alimentar). O ganho de peso e oconsumo de ração foram afetados pelos tratamentos $(\mathrm{P}<0,05)$, não havendo efeito para conversão alimentar, rendimento de carcaça e cortes nobres $(\mathrm{P}>0,05)$. Foram observadas diferenças $(\mathrm{P}<0,05)$ no sangue para os níveis de albumina, onde a inclusão de $12 \%$ demonstrou menores níveis.O menor custo com alimentação por $\mathrm{kg}$ de GP foi obtido com a ração com $6 \%$ de inclusão da FMB, o que resultou na maior margem bruta. Concluiu-se que a farinha do mesocarpo do babaçu é técnica e economicamente viável em até $6 \%$ em rações para frangos de corte de 22 a 42 dias de idade.

Palavras chave: albumina, alimento alternativo, coproduto; nutrição

\section{SUMMARY}

The work was to evaluate the technical and economic feasibility of including the inclusion of the babassumesocarp flour in diets for broilers from 22 to 42 days old. 80 broilers, male Cobb $500^{\circledR}$ line were distributed in a completely randomized design with four treatments $(0,6,12$ and $18 \%$ inclusion flour babassumesocarp) and five replicates and each plot were used consisting of 4 birds. At 42 days of age the birds and the experimental diets were weighed for obtaining performance (feed intake, weight gain and feed conversion). Weight gain and feed intake were affected by treatments $(\mathrm{P}<$ $0.05)$, with no effect on feed conversion, carcass yield and prime cuts $(\mathrm{P}>0.05)$. Differences $(\mathrm{P}<$ $0.05)$ on blood levels of albumin, where the addition of $12 \%$ showed lower levels were observed. The lowest feed cost per kg GP was obtained in the diet with $6 \%$ inclusion of FMB, which resulted in higher gross margin. It was concluded that the flour from babassumesocarp is technically and economically feasible in up to $6 \%$ in diets for broilers from 22 to 42 days old.

Keyword: albumin, alternative food, byproduct, nutrition 
Rev. Bras. Saúde Prod. Anim., Salvador, v.16, n.1, p.223-231 jan./mar., 2015 http://www.rbspa.ufba.br ISSN 15199940

\section{INTRODUÇÃO}

A avicultura de corte se sobressai como uma das atividades econômicas mais importantes na estrutura agropecuária brasileira, sendo responsávelpela evolução da cadeia produtiva, o melhoramento genético, a nutrição e o manejo (TAVERNARI et al., 2008a).

Entretanto, um dos problemas enfrentados pelos avicultores são as oscilações do preço do milho no mercado, que acabam onerando os custos de produção nas diferentes regiões do país (CARNEIRO et al., 2009; BASTOS et al., 2007; RAMOS et al., 2006; BRUNELLI et al., 2006; NASCIMENTO et al., 2005).

O emprego de alimentos alternativos tem se tornado expressivo nos últimos anos, fazendo com que nutricionistas pesquisem novos alimentos, com $\mathrm{o}$ intuito de reduzir custos na alimentação animal (CANCHERINI et al., 2005), dentre eles o coco de babaçu.

$\mathrm{O}$ processo de extração dococo do babaçu para a produção de óleo, resulta em alguns coprodutos, como a farinha do mesocarpo do babaçu. Pesquisas estão sendo desenvolvidas com sua utilização desse coproduto na alimentação anima.1 e tem demonstrado viabilidade de uso.

Cruz et al. (2013) avaliaram os efeitos de níveis crescentes de inclusão da farinha do mesocarpo do babaçu $(0 ; 6$; 12 e 18\%) sobre o consumo de ração, ganho de peso, conversão alimentar, rendimento de carcaça e proteínas do sangue de frangos de corte na fase de 8 a 21 dias de idade e verificaram que a adição de até $12 \%$ de farinha do mesocarpo de babaçu não comprometeu o desempenho.

Trabalhos com a inclusão da farinha do mesocarpo do babaçu em rações de frangos de corte na fase de crescimento/terminação são escassos, diante disso, objetivou-se com este trabalho avaliar a viabilidade técnica e econômica da inclusão da inclusão da farinha do mesocarpo do babaçu em dietas de frangos de corte de 22 a 42 dias de idade.

\section{MATERIAL E MÉTODOS}

O experimento foi conduzido no Setor de Avicultura da Universidade Federal do Tocantins, Araguaína - TO, no período de 31 de março a 20 de abril de 2011. Foi utilizado um galpão convencional disposto no sentido LesteOeste, coberto com palha de babaçu, com piso de concreto dotado de gaiolas com área de $1 \mathrm{~m}^{2}$.

Foram utilizados 80 frangos machos, da linhagem Cobb $500^{\circledR}$, os quais foram alimentados com ração formulada para atender suas exigências nutricionais de acordo com Rostagno et al.(2005) até os 21 dias de vida. No $22^{\circ}$ dia iniciou-se o experimento, com as aves apresentando peso médio de $719 \mathrm{~g}$,distribuídas em delineamento experimental inteiramente casualizado com quatro tratamentos $(0 ; 6$; 12 e $18 \%$ de inclusão de farinha do mesocarpo de babaçu (FMB) e cinco repetições, sendo cada unidade experimental composta por quatro aves.

As gaiolas eram dotadas comedouro e bebedouro tipo calha. A limpeza dos bebedouros e comedouros e $\mathrm{O}$ reabastecimento de água e ração foram realizados duas vezes ao dia, possibilitando a ingestão de água e o consumo de ração ad libitum.

As condições ambientais no interior das instalações foram registradas diariamente seis vezes ao dia $(8: 00 ; 10: 00 ; 12: 00$; 14:00; 16:00 e 18:00h), por meio de termômetros de máxima e mínima, bulbo seco, bulbo úmido e globo negro, sendo estes valores convertidos em ITGU 
Rev. Bras. Saúde Prod. Anim., Salvador, v.16, n.1, p.223-231 jan./mar., 2015 http://www.rbspa.ufba.br ISSN 15199940

(Índice de Temperatura Globo e Umidade), de acordo com Buffington et al. (1981).

$\mathrm{O}$ valor da temperatura média de globo negro e umidade relativa média do ar no interior do galpão, durante o período experimental foi de $29,7 \pm 1,4^{\circ} \mathrm{C}$ e $60 \%$, respectivamente, equivalendo ao ITGU de $76,5 \pm 1,5$.
A ração basal foi formulada a base de milho e farelo de soja (0\% de inclusão de farinha do mesocarpo de babaçu (FMB)). As rações experimentais foram formuladas de acordo com Rostagno et al. (2005) para conterem $3.150 \mathrm{kcal} / \mathrm{kg}$ de energia metabolizável e $20 \%$ de proteína bruta (Tabela 1).

Tabela 1. Composição das rações experimentais contendo níveis crescentes de farinha do mesocarpo do babaçu para frangos de 22 a 42 dias de idade

\begin{tabular}{|c|c|c|c|c|}
\hline \multirow{2}{*}{ Ingredientes } & \multicolumn{4}{|c|}{ Níveis de inclusão da Farinha do Mesocarpo do babaçu (\%) } \\
\hline & 0 & 6 & 12 & 18 \\
\hline Milho & 64,84 & 56,02 & 47,21 & 38,40 \\
\hline Farelo de Soja (45\%) & 29,10 & 30,38 & 31,66 & 32,95 \\
\hline Farinha mesocarpo Babaçu & 0,00 & 6,00 & 12,00 & 18,00 \\
\hline Fosfato bicálcico & 1,55 & 1,58 & 1,60 & 1,63 \\
\hline Óleo de soja & 2,82 & 4,37 & 5,92 & 7,46 \\
\hline Calcário & 0,72 & 0,67 & 0,62 & 0,57 \\
\hline Sal comum & 0,40 & 0,40 & 0,41 & 0,41 \\
\hline DL-Metionina & 0,22 & 0,23 & 0,25 & 0,27 \\
\hline L-Lisina $\mathrm{HCl}$ & 0,23 & 0,21 & 0,196 & 0,17 \\
\hline L-Treonina & 0,05 & 0,05 & 0,06 & 0,07 \\
\hline Núcleo ${ }^{1}$ & 0,04 & 0,04 & 0,04 & 0,04 \\
\hline Total & 100,00 & 100,00 & 100,00 & 100,00 \\
\hline \multicolumn{5}{|c|}{ Composição nutricional calculada } \\
\hline EM (kcal/kg) & 3,15 & 3,15 & 3,15 & 3,15 \\
\hline Cálcio (\%) & 0,79 & 0,79 & 0,79 & 0,79 \\
\hline Fósforo disponível (\%) & 0,39 & 0,39 & 0,39 & 0,39 \\
\hline Proteína bruta (\%) & 19,20 & 19,20 & 19,20 & 19,20 \\
\hline Lisina total $(\%)$ & 1,15 & 1,15 & 1,15 & 1,15 \\
\hline Metionina + cistina total $(\%)$ & 0,82 & 0,82 & 0,82 & 0,82 \\
\hline Sódio (\%) & 0,19 & 0,19 & 0,19 & 0,19 \\
\hline Treonina total (\%) & 0,78 & 0,78 & 0,78 & 0,78 \\
\hline Fibra bruta & 3,22 & 5,30 & 7,38 & 9,46 \\
\hline
\end{tabular}

As variáveis avaliadas foram consumo de ração (CR), ganho de peso (GP), conversão alimentar (CA), rendimentos de carcaça, cortes nobres (peito, coxa e sobrecoxa), proteínas plasmáticas do sangue e o custo da alimentação por $\mathrm{kg}$ de frango produzido.

O consumo de ração foi calculado pela diferença entre o fornecido e as sobras, sendo o ganho de peso calculado pela diferença entre o peso das aves no início e no final do experimento. A partir dos dados de consumo de ração e ganho de peso calculou-se a conversão alimentar (CR/GP).

No $42^{\circ}$ dia duas aves de cada unidade experimental, foram escolhidas ao acaso ambas, com peso corporal próximo a 
Rev. Bras. Saúde Prod. Anim., Salvador, v.16, n.1, p.223-231 jan./mar., 2015 http://www.rbspa.ufba.br ISSN 15199940

média da parcela $( \pm 5 \mathrm{~g})$, totalizando 40 aves, foram abatidas, após jejum alimentar de 12 horas e evisceradas para avaliação dos pesos relativos $(\%)$ das carcaças inteiras (com pés, pescoço e cabeça) e dos cortes nobres (coxa, sobrecoxa e peito com osso).

$\mathrm{O}$ rendimento da carcaça eviscerada foi calculado em relação ao peso vivo após jejum, enquanto os rendimentos dos cortes foram calculados em relação ao peso absoluto da carcaça.

No decorrer do abate no momento da sangria, foi coletado sangue de duas aves de cada parcelasendo encaminhado para o Laboratório de Patologia Clínica da Universidade Federal do Tocantins, onde foi centrifugado a 3000rpm durante 15 minutos para a obtenção do soro. Posteriormente foram avaliados: as proteínas totais, albumina $\mathrm{e}$ globulinas sendo esta última obtida pela diferença entre a concentração das proteínas totais e albumina, com auxílio de técnicas foto colorimétricas, utilizando kits comerciais de reagentes. Todas as variáveis de desempenho, rendimento de carcaça e proteínas sanguíneas foram submetidos aos testes de normalidade (Cramer Von Mises) e Homocedasticidade (Levene).

As análises estatísticas foram realizadas com o auxílio do software SAS 9.1 (STATISTICAL ANALYZES SYSTEM, 2013). E as médias de cada tratamento também foram comparadas por meio do teste SNK (Student Newman Keuls). Nas variáveis cujos efeitos da inclusão da farinha do mesocarpo do babaçu foram detectados pela análise de variância, realizaram-se análises de regressão por meio de modelos polinomiais.

Determinou-se o custo com alimentação por kg de frango produzido como segue:

$$
\mathrm{R} \$ / \mathrm{kgGPi}=(\mathrm{CRi} \times \mathrm{R} \$ \mathrm{Ri}) / \mathrm{GPi} \text {; com } \mathrm{i}=1,2,3,4 .
$$

Em que: $\mathrm{R} \$ / \mathrm{kgGPi}=$ custo por $\mathrm{kg}$ de frango $(\mathrm{R} \$ / \mathrm{kg})$ produzido com a utilização do i-ésimo nível de inclusão da farinha do mesocarpo do babaçu; Cri = quantidade de ração $(\mathrm{kg})$ consumida no i-ésimo nível de inclusão da farinha do mesocarpo do babaçu; $\mathrm{R} \$ \mathrm{Ri}=$ custo da ração $(\mathrm{R} \$ / \mathrm{kg})$ contendo o i-ésimo nível de inclusão da farinha do mesocarpo do babaçu; GPi = ganho de peso $(\mathrm{kg})$ das aves que receberam o iésimo nível inclusão da farinha do mesocarpo do babaçu.

A margem por $\mathrm{kg}$ de frango para cada nível de inclusão da farinha do mesocarpode babaçu nas rações foi calculada pela expressão:

$\mathrm{Mi}=\mathrm{PVF}-\mathrm{R} \$ / \mathrm{kgGPi}$

Em que: $\mathrm{Mi}=$ margem por $\mathrm{kg}$ de frango ( R\$) obtida com a utilização do i-ésimo nível de inclusão da farinha do mesocarpo do babaçu; $\mathrm{PVF}=$ preço de venda do frango vivo ( $\mathrm{R} \$ / \mathrm{kg})$;
$\mathrm{R} \$ / \mathrm{kgGP}=$ custo por $\mathrm{kg}$ de frango $(\mathrm{R} \$ / \mathrm{kg}$ ) produzido com a utilização do i-ésimo nível de inclusão da farinha do mesocarpo do babaçu.

\section{RESULTADOS E DISCUSSÃO}

A Farinha do mesocarpo de babaçu apresentou $3.689 \mathrm{kcal} / \mathrm{kg}$ de EB (Tabela 2 ), valor similar ao encontrado por Silva (2010), de $3.687 \mathrm{kcal} / \mathrm{kg}$ e inferior ao observado por Santos Neta (2010), que ao analisar a farinha do mesocarpo em diferentes épocas do ano (chuvosa e seca), encontrou valores de $4.221 \mathrm{e}$ $4.178 \mathrm{kcal} / \mathrm{kg}$, respectivamente.

O percentual de proteína bruta encontrado foi de $3,06 \%$, próximo do valor encontrado por Silva (2010) de $1,91 \%$, sendo caracterizadocomo um alimento pobre em proteína. Possivelmente, essas diferenças estão 
Rev. Bras. Saúde Prod. Anim., Salvador, v.16, n.1, p.223-231 jan./mar., 2015 http://www.rbspa.ufba.br ISSN 15199940

relacionadasàs diferentes condições de processamento, solo, época do ano e espécie da planta.
Os níveis de inclusão da FMB nas rações afetaram $(\mathrm{P}<0,05)$, o consumo de ração (CR) e o ganho de peso (GP), não havendo efeito $(\mathrm{P}>0,05)$ sobre conversão alimentar (CA) (Tabela 3 ).

Tabela 2. Composição dos ingredientes utilizados na formulação das rações experimentais (com base na matéria natural)

\begin{tabular}{lccc}
\hline Nutriente & Milho $^{1}$ & Farelo de Soja $^{1}$ & FMB \\
\hline Fibra Bruta (\%) & 2,60 & 5,30 & $37,33^{2}$ \\
Energia Bruta (kcal/kg) & 3.381 & 2.256 & $3.689^{2}$ \\
Proteína bruta (\%) & 8,28 & 45,32 & $3,062^{2}$ \\
Cálcio (\%) & 0,030 & 0,240 & $0,10^{2}$ \\
Fósforo disponível (\%) & 0,080 & 0,180 & $\mathrm{ND}$ \\
Cloro (\%) & 0,050 & 0,050 & $\mathrm{ND}$ \\
Potássio (\%) & 0,280 & 1,830 & $\mathrm{ND}$ \\
Sódio (\%) & 0,020 & 0,020 & $\mathrm{ND}$ \\
Lisina total (\%) & 0,240 & 2,770 & $\mathrm{ND}$ \\
Metionina total (\%) & 0,170 & 0,640 & $\mathrm{ND}$ \\
Met+cistina total (\%) & 0,360 & 1,370 & $\mathrm{ND}$ \\
Treonina total $(\%)$ & 0,320 & 1,780 & $\mathrm{ND}$ \\
Triptofano total $(\%)$ & 0,070 & 0,620 & $\mathrm{ND}$ \\
\hline
\end{tabular}

${ }^{1}$ Rostagno et al. (2005).

${ }^{2}$ Análises realizadas no Laboratório de Nutrição Animal do Centro de Ciência Animal Tropical da Universidade Federal do Tocantins.

FMB = farinha do mesocarpo do babaçu.

Tabela 3. Médias de consumo de ração $(\mathrm{kg})$, ganho de peso $(\mathrm{kg})$ e conversão alimentar $(\mathrm{kg} / \mathrm{kg})$ de frangos de corte de 22 a 42 dias de idade, de acordo com o nível de inclusão de farinha de babaçu

\begin{tabular}{|c|c|c|c|c|c|c|}
\hline \multirow{2}{*}{ Variáveis } & \multicolumn{4}{|c|}{ Níveis de inclusão de farinha de babaçu (\%) } & \multirow{2}{*}{$P>0,05$} & \multirow{2}{*}{$\mathrm{CV}(\%)$} \\
\hline & $0 \%$ & $6 \%$ & $12 \%$ & $18 \%$ & & \\
\hline CR & $2,91^{\mathrm{a}}$ & $2,69^{b}$ & $2,59^{b}$ & $2,62^{b}$ & 0,02 & 5,14 \\
\hline GP & $1,42^{\mathrm{a}}$ & $1,35^{\mathrm{ab}}$ & $1,27^{\mathrm{bc}}$ & $1,20^{\mathrm{c}}$ & 0,09 & 4,95 \\
\hline $\mathrm{CA}$ & 2,03 & 1,98 & 2,4 & 2,18 & 0,11 & 5,71 \\
\hline
\end{tabular}

a,b,c Médias seguidas de letras iguais não diferem pelo teste SNK $(\mathrm{P}>0,05)$.

$\mathrm{CV}$ = coeficiente de variação (\%); Teste $\mathrm{F}$ da análise de variância.

$\mathrm{CR}=$ consumo de ração; $\mathrm{GP}=$ ganho de peso; $\mathrm{CA}$ = conversão alimentar.

Houve efeito quadrático da inclusão da FMB sobre o $\mathrm{CR}=2,9109-0,047 \mathrm{FMB}$ $+0,0017 \mathrm{FMB}^{2}\left(\mathrm{P}<0,001, \mathrm{R}^{2}=0,749\right)$ (Figura 1). A equação apontou um ponto de mínimo de $13,8 \%$ de inclusão para essa variável.Os níveis de fibra podem ter influenciado essa variável, fazendo com que o consumo fosse reduzido nos tratamentos com inclusão da farinha.

Observou-se que a inclusão da FMB promoveu redução linear $(\mathrm{P}<0,01)$ no ganho de peso, segundo a equação: GP $=1,4317-0,0128$ FMB $\left(\mathrm{R}^{2}=0,9987\right)$, (Figura 2). O aumento nos níveis de fibra nas rações experimentais $(3,22$; 
Rev. Bras. Saúde Prod. Anim., Salvador, v.16, n.1, p.223-231 jan./mar., 2015 http://www.rbspa.ufba.br

5,$30 ; 7,38$ e 9,46\%), resultantes dos níveis crescentes de inclusão da FMB $(0,6,12$ e $18 \%)$, podem ter influenciado no GP das aves. De acordo com Brito et al. (2008) e Tavernari et al. (2008b) a baixa digestibilidade da fibra, podem reduzir a energia dos alimentos e prejudicar a utilização de outros nutrientes.

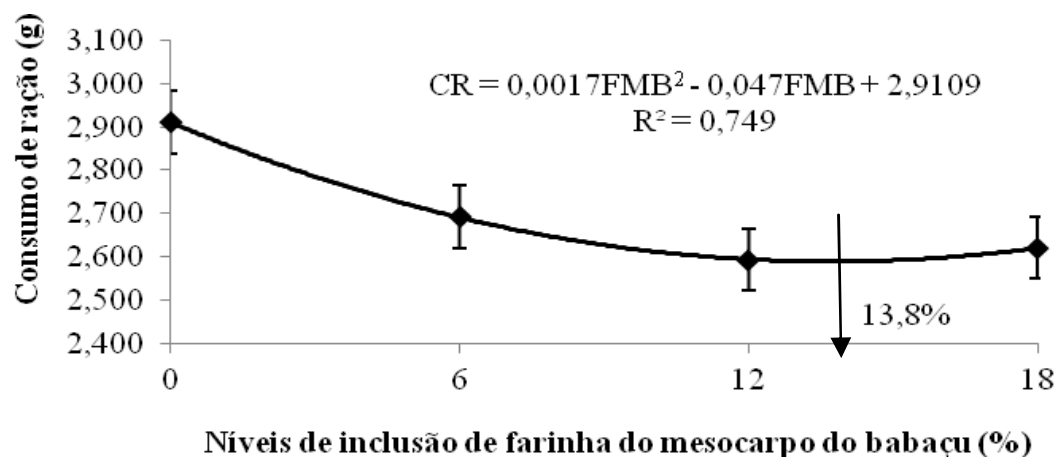

Figura 1. Níveis de farinha do mesocarpo de babaçu (\%) sobre o consumo de ração de frangos de corte na fase de 22 a 42 dias de idade

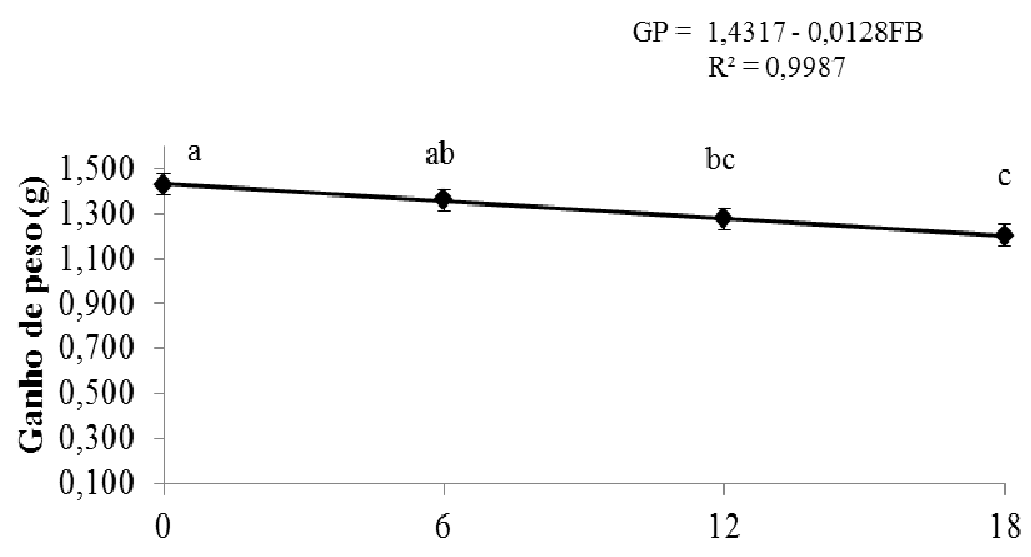

Níveis de inclusão da farinha do mesocarpo do babaçu (\%)

Figura 2. Níveis de farinha do mesocarpo de babaçu (\%) sobre o ganho de peso de frangos de corte na fase de 22 a 42 dias de idade

Os rendimentos de carcaça e cortes nobres não foram influenciados ( $\mathrm{P}>0,05)$, pela inclusão da farinha nas rações (Tabela 4). Nota-se, que embora o ganho de peso tenha piorado com o aumento dos níveis, tanto o rendimento da carcaça quanto os cortes nobres foram proporcionais não sendo evidenciada uma piora nesses rendimentos.

Embora trabalhos avaliando a FMB para frangos de corte sejam escassos, esse comportamento de rendimento de carcaça e cortes nobres, já foram encontrados em frangos submetidos à inclusão de subprodutos como relata Cancherini et al. (2005) que analisaram 
Rev. Bras. Saúde Prod. Anim., Salvador, v.16, n.1, p.223-231 jan./mar., 2015 http://www.rbspa.ufba.br ISSN 15199940

o subprodutos do arroz (farelo de arroz integral e quirera) e da mesma forma, não evidenciaram interferência dos subprodutos avaliados sobre os rendimentos de carcaça e cortes nobres (peito, coxa e sobrecoxa).

Para os dados de proteínas plasmáticas do sangue de frangos de corte aos 42 dias de idade, alimentados com FMB (Tabela 5), não foram detectadas diferenças $(\mathrm{P}>0,05)$ para os níveis de proteína total e globulinas. No entanto, os níveis de inclusão de FMB nas rações, afetaram $(\mathrm{P}>0,05)$ os níveis de albumina, entre o tratamento controle $(2,60 \mathrm{~g} / \mathrm{dL})$ e o nível de inclusão de $12 \%$ de FMB $(1,81 \mathrm{~g} / \mathrm{dL})$. A equação que representa esse efeito é: $\mathrm{ALB}=2,6154-0,1251+$ $0,005 \mathrm{FMB}^{2}\left(\mathrm{P}<0,001, \mathrm{R}^{2}=0,9949\right)$.

Tabela 4. Médias do rendimento de carcaça (\%) e pesos relativos dos cortes nobres de frangos de corte aos 42 dias alimentados com diferentes níveis de farinha do mesocarpo de babaçu

\begin{tabular}{lllllll}
\hline \multirow{2}{*}{ Variável } & \multicolumn{6}{c}{ Níveis de inclusão de farinha de babaçu (\%) } \\
\cline { 2 - 7 } & 0 & 6 & 12 & 18 & $\mathrm{CV}$ & $\mathrm{P}^{2}$ \\
\hline Rendimento de carcaça (\%) & 80,07 & 80,91 & 80,62 & 80,17 & 0,78 & 0,53 \\
Coxa (\%) & 12,16 & 12,63 & 11,99 & 12,24 & 5,05 & 0,50 \\
Sobrecoxa (\%) & 14,22 & 13,90 & 14,72 & 14,43 & 5,50 & 0,47 \\
Peito (\%) & 29,87 & 30,43 & 30,74 & 31,37 & 3,97 & 0,31 \\
\hline
\end{tabular}

${ }^{\mathrm{I}}$ Coeficiente de variação $(\%) ;{ }^{2}$ Teste $\mathrm{F}$ da análise de variância.

Tabela 5. Valores bioquímicos da proteína total, albumina e globulinas em g/ dL de frangos de corte aos 42 dias de idade

\begin{tabular}{|c|c|c|c|c|c|c|}
\hline \multirow{2}{*}{ Variáveis } & \multicolumn{4}{|c|}{ Níveis de farinha de babaçu } & \multirow{2}{*}{$\mathrm{P}>0,05$} & \multirow{2}{*}{$\mathrm{CV}(\%)$} \\
\hline & $0 \%$ & $6 \%$ & $12 \%$ & $18 \%$ & & \\
\hline Proteína total & $4,97^{\mathrm{a}}$ & $4,95^{\mathrm{a}}$ & $5,12^{\mathrm{a}}$ & $5,31^{\mathrm{a}}$ & 0,89 & 16,62 \\
\hline Albumina & $2,60^{\mathrm{a}}$ & $2,07^{\mathrm{ab}}$ & $1,81^{b}$ & $2,00^{\mathrm{ab}}$ & 0,04 & 19,37 \\
\hline Globulinas & $2,36^{\mathrm{a}}$ & $2,87^{\mathrm{a}}$ & $3,31^{\mathrm{a}}$ & $3,31^{\mathrm{a}}$ & 0,35 & 31,40 \\
\hline
\end{tabular}

${ }^{\mathrm{a}, \mathrm{b}}$ Médias seguidas de letras iguais não diferem pelo teste $\mathrm{SNK}(\mathrm{P}>0,05)$.

$\mathrm{CV}=$ coeficiente de variação $(\%) ;{ }^{2}$ Teste $\mathrm{F}$ da análise de variância.

O nível de albumina no sangue dos frangos alimentados com ração contendo $12 \%$ de inclusão da FMB, apresentou-se fora da faixa de referência de 2,5g/dL (LUMEIJ, 1997; KANEKO, 1997), considerada normal para frangos de corte. A alteração desse nível pode ser justificada pelo estresse pré abate, pois, quadro de desidratação pode modificar a concentração da albumina sanguínea (CAMPBELL, 2004).

$\mathrm{O}$ menor custo com alimentação por $\mathrm{kg}$ de GP foi obtido com a dieta contendo
6\% de inclusão da FMB, o que resultou na maior margem bruta em relação à alimentação (Tabela 6). Considerando os preços dos insumos utilizados na fabricação das rações experimentais, observou-se que a inclusão de níveis crescentes da FMB promoveu aumento linear no preço das rações, consequência, principalmente, do aumento dos níveis de óleo de soja à medida que os níveis de FMB aumentaram (Tabela 1). 
Rev. Bras. Saúde Prod. Anim., Salvador, v.16, n.1, p.223-231 jan./mar., 2015 http://www.rbspa.ufba.br ISSN 15199940

Tabela 6. Dados econômicos obtidos com frangos de corte de 22 a 42 dias de idade recebendo farinha de babaçu

\begin{tabular}{|c|c|c|c|c|}
\hline \multirow{2}{*}{ Variáveis } & \multicolumn{4}{|c|}{ Níveis de farinha do mesocarpo de babaçu (\%) } \\
\hline & 0 & 6 & 12 & 18 \\
\hline Custo da alimentação ${ }^{1}(\mathrm{R} \$ / \mathrm{kg})$ & 0,93 & 0,95 & 0,97 & 0,98 \\
\hline Custo da alimentação por kg de GP (R $\$ / k g)$ & 1,90 & 1,89 & 1,97 & 2,15 \\
\hline Margem bruta $^{2}(\mathrm{R} \$ / \mathrm{kg})$ & 0,95 & 0,97 & 0,88 & 0,70 \\
\hline
\end{tabular}

${ }^{1}$ Considerando os seguintes preços: milho $=\mathrm{R} \$ 0,59 / \mathrm{kg}$; farelo de soja $=\mathrm{R} \$ 1,30 / \mathrm{kg}$; farinha do mesocarpo de babaçu $=\mathrm{R} \$ 0,22 / \mathrm{kg}$; fosfato bicálcico $=\mathrm{R} \$ 2,77 / \mathrm{kg}$; óleo de soja $=\mathrm{R} \$ 2,49 / \mathrm{kg}$; calcário = $\mathrm{R} \$ 0,43 / \mathrm{kg} ; \mathrm{sal}=0,80 / \mathrm{kg} ;$ DL-metionina $=\mathrm{R} \$ 11,87 / \mathrm{kg} ; \mathrm{L}$-lisina $\mathrm{HCl}=\mathrm{R} \$ 6,67 / \mathrm{kg} ;$ L-treonina $=$ $\mathrm{R} \$ 6,76 / \mathrm{kg}$; Suplemento mineral e vitamínico $=\mathrm{R} \$ 9,00 / \mathrm{kg}$.

${ }^{2}$ Considerando o preço do frango vivo de $\mathrm{R} \$ 2,85 / \mathrm{kg}$.

Os resultados do presente estudo evidenciaram que a inclusão de $6 \%$ da FMB, em dietas de frangos de corte de 22 a 42 dias de idade, é técnica e economicamente viável, pois a inclusão desse nível resultou em desempenho semelhante à dieta padrão (com milho e farelo de soja, sem FMB), menor custo com ração por $\mathrm{kg}$ de GP e maior margem bruta em relação à alimentação.

\section{REFERÊNCIAS}

BASTOS, S.C.; FUENTES, M.F.F.; FREITAS, E.R.; ESPÍNDOLA, G.B.; BRAGA, C.V.P. Efeito da inclusão do farelo de coco em rações para frangos de corte. Revista Ciência Agronômica, v.38, n.3, p.297-303, 2007.

BRITO, M.S; OLIVEIRA, C.F.S; SILVA, T.R.G; LIMA, R.B; MORAIS, S.N; SILVA, J.H.V. Polissacarídeos não amiláceos na nutrição de monogástricos revisão. Revista Acta Veterinária Brasilica, v.2, n.4, p.111-117, 2008.

BRUNELLI, S.R.; PINHEIRO, J.W.; SILVA, C.A.da; FONSECA, N.A.N.; OLIVEIRA, D.D.de; CUNHA, G.E.; SOUZA, L.F.A.de. Inclusão de farelo de gérmen de milho desengordurado na alimentação de frangos de corte. Revista Brasileira Zootecnia,v.35, n.4, p.13491358, 2006.

BUFFINGTON, D. E.; COLLAZOAROCHO, A.; CANTON, G. H.; PITT, D.; THATCHER, W. W.; COLLIER, R. J. Black globe humidity index (BGHI) as comfort equation for dairy cows.

Transactions of the ASAE, v.24, n.3, p.711-714, 1981.

CAMPBELL, T. Blood biochemistry of lower vertebrates.In: 55th ANNUAL MEETING OF THE AMERICAN COLLEGE OF VETERINARY PATHOLOGISTS, $55 \&$ 39, 2004, Middleton WI, USA.Proccending....Middleton WI, USA:American College of Veterinary Pathologists / American Society for Veterinary Clinical Pathology,2004.

CARNEIRO, A.P.M.; PASCOAL, L.A.F.; WATANABE, P.H.; SANTOS, I.B.; LOPES, J.M.; ARRUDA, J.C.B. de. Farelo de babaçu em rações para frangos de corte na fase final: desempenho, rendimento de carcaça e avaliação econômica. Ciência Animal Brasileira, v.10, n.1, p.40-47, 2009. 
Rev. Bras. Saúde Prod. Anim., Salvador, v.16, n.1, p.223-231 jan./mar., 2015 http://www.rbspa.ufba.br ISSN 15199940

CHACHERINI, L.C; JUNQUEIRA, O.M; OLIVEIRA, M.C; ANDRIOTTI, M.O; BARBOSA, M.J.B. Utilização de subprodutos de origem animal em dietas formuladas com base na proteína bruta e proteína ideal para frangos de corte de 22 a 42 dias de idade.Revista Brasileira de Zootecnia,v.34, n.2 p.525-540, 2005.

CRUZ, L.A.; VAZ, R.G.M.V.; SIQUEIRA, J.C.; SILVA, G.F.1C; RODRIGUES, K.F.; STRINGHINI, J.H.; RAMOS, A.T.; DE SOUSA, J.P.1G; CALIXTO, M. S.; FONSECA, F.L.R. Farinha do mesocarpo do babaçu em rações de frangos de corte na fase inicial. Archivos de Zootecnia, v.62, n.240, p.525-532. 2013.

KANEKO, J.; HARVEY, J.; BRUSS, M. Clinical biochemistry of domestic animals.5th ed. San Diego, Academic Press, 1997. 932p.

LUMEIJ, J.T. Avian Clinical Biochemistry. In: KANEKO, J.J.; HARVEY, J.W.; BRUSS, M.L. (Eds.). Clinical biochemistry of domestic animals.5th ed. San Diego, Academic Press, 1997.932p.

NASCIMENTO, G.A.J. do; COSTA, F.G.P.; AMARANTE JÚNIOR, V.S.; BARROS, L.R. Efeitos da substituição do milho pela raspa de mandioca na alimentação de frangos de corte, durante as fases de engorda e final. Ciência Agrotecnologia, v.29, n.1, p.200-207, 2005.

RAMOS, L,S.N.; LOPES, J.B.; FIGUEIRÊDO, A.V.; FREITAS, A.C.; FARIAS, L.A.; SANTOS, L.S.; SILVA, H.O. Polpa de caju em rações para frangos de corte na fase final: desempenho e características de carcaça. Revista Brasileira de Zootecnia, v.35, n.3, p.804-810, 2006.
ROSTAGNO, H.S; ALBINO, L.F.T; DONZELE, J.L; GOMES, P.C; OLIVEIRA, R.F. de; LOPES, D.C; FERREIRA, A.S; BARRETO, S.L. de $T$. Tabelas brasileiras para aves $\mathrm{e}$ suínos: composição de alimentos e exigências nutricionais. Viçosa: Universidade Federal de Viçosa, 2005. $186 \mathrm{p}$.

SANTOS NETA, E.R. Avaliação de subprodutos do babaçu (Palmaeorbignyamartiana) na alimentação de frangos de corte. 2010. 59 p. Dissertação (Mestrado em Ciência Animal Tropical) - Escola de Medicina Veterinária e Zootecnia, Universidade Federal do Tocantins, Araguaína.

SILVA, E.A.Valores de energia metabolizável e de aminoácidos digestíveis de alguns alimentos. 2010. 77p. Dissertação (Mestrado em Zootecnia) - Universidade Federal de Viçosa, Viçosa.

STATISTICAL ANALYSIS SYSTEM.SAS: user's guide. Version 9.1 ed. Cary, USA: SAS InstituteInc, USA, 2003.

TAVERNARI, F.C.; ALBINO, L.F.T.;DUTRAJUNIOR, W.M.; LELIS, G.R.;NERY, L.R.; MAIA, R.C. Farelo de girassol: composição e utilização na alimentação de frangos de corte.

Revista Eletrônica Nutritime,v.5, n.5, p.638-647, 2008a.

TAVERNARI, F.C.; CARVALHO, T.A.; ASSIS, A.P.; LIMA, H.J.D'A. Polissacarídeo não amiláceo solúvel na dieta de suínos e aves. Revista Eletrônica Nutritime, v.5, n.5, p.673689, 2008b.

Data de recebimento: 20/06/2014

Data de aprovação: 23/02/2015 